\title{
Primary Anorectal Melanomas Interest of Targeting C-KIT in Two Cases from a Series of 11 Patients
}

\author{
Karima Oualla ${ }^{*}$, Nawfel Mellas ${ }^{1}$, Fatimazahra El'mrabet ${ }^{1}$, Samia Arifi' ${ }^{1}$, Afaf Amarti², \\ Khalid Ait Taleb ${ }^{3}$, Siham Tizniti4 ${ }^{4}$ Omar Elmesbahi ${ }^{1}$ \\ ${ }^{1}$ Department of Medical Oncology, Hassan II University Hospital, Fez, Morocco \\ ${ }^{2}$ Department of Pathology, Hassan II University Hospital, Fez, Morocco \\ ${ }^{3}$ Department of Visceral Surgery, Hassan II University Hospital, Fez, Morocco \\ ${ }^{4}$ Department of Radiology, Hassan II University Hospital, Fez, Morocco \\ Email: ${ }^{*}$ karimarauf@hotmail.com
}

Received 23 November 2013; revised 23 December 2013; accepted 2 January 2014

Copyright (C) 2014 by authors and Scientific Research Publishing Inc.

This work is licensed under the Creative Commons Attribution International License (CC BY).

http://creativecommons.org/licenses/by/4.0/

(c) (i) Open Access

\section{Abstract}

Background: The anorectal location of melanomas is extremely rare $(1 \%-3 \%$ of all melanomas), and the prognosis remains poor because of the aggressiveness and the high metastatic potential of those tumors. The discovery that the KIT oncogene may be aberrantly activated in a subset of patients with anorectal melanoma creates a realm of possibility for the development of targeted molecular therapy. Aim: to show the epidemiologic, clinico-radiological, histological features and treatment management especially in patients with over-expression of C-KIT treated by Imatinib. Methods: It is a retrospective study conducted in the department of medical oncology at Hassan II University Hospital between January 2007 and January 2012, including all patients with histologically proven melanoma of the anorectal area. Results: 11 cases were collected, with slight female predominance. Nine patients were metastatic at the moment of diagnosis, and only two with local stage, but evolution was marked by local and distant recurrence less than 12 months after abdominoperineal resection. First line of chemotherapy was based mainly on paclitaxel, carboplatine and dacarbazine. Response was modest with only 3 partial responses, 4 patients with disease stability, and 4 patients with disease progression. Two patients, with over expression of C-KIT, received Imatinib as second line of treatment with significant improvement of symptoms and radiological response reaching $50 \%$. Seven patients died with a median survival of 11 months from diagnosis to the date of death. Conclusion: Primary anorectal melanomas are very rare, with high aggressiveness and poor prognosis. Treatment management is still a big challenge given to the modest efficacy of conventional chemotherapy. Better understanding of carcinogenesis and signaling pathways will allow development of new targeted therapies.

\footnotetext{
*Corresponding author.
} 


\section{Keywords}

\section{Melanoma; Anorectal Area; Surgery; Chemotherapy; C-KIT; Imatinib}

\section{Introduction}

Primary malignant melanoma of the anus and rectum is a rare and highly lethal neoplasm. Mucosal melanomas account for approximately $1.2 \%$ of all melanomas, and anorectal melanomas account for fewer than $25 \%$ of all mucosal melanomas [1], and approximately $1 \%$ of cancers afflicting this particular area [2]. The most frequent presentation symptom is rectal bleeding followed by change in defecatory habits and proctalgia [2].

Prognosis is very poor especially for advanced stages. Only $10 \%$ of all patients will be alive at 5 years and nearly $60 \%$ of patients present with metastatic disease [3].

\section{Methods}

It is a retrospective study conducted in the department of medical oncology at Hassan II University Hospital between January 2007 and January 2012, including all adult patients with histologically proven melanoma of the anorectal area.

\section{Results}

Eleven patients were collected, 6 men and 5 women, the mean age was 61 years (50 - 81 ans). The symptoms were mainly rectal bleeding, proctalgia, rectal syndrome, and transit disorders. Colonoscopy was performed in all cases and revealed a circumferential non-pigmented burgeoning tumor in all cases and stenotic tumor in 8 cases. The radiological assessment included a thoraco-abdomino-pelvic scan in all cases and 4 patients underwent a pelvic MRI (Figure 1).

Nine patients had a metastatic disease at the diagnosis, all in the liver (Figure 2), lymph node and lung. Only two patients were not metastatic but with locally advanced disease, and proceeded to surgery, they underwent an abdomino-perineal resection with lymphadenectomy; but the evolution was marked by occurrence of pulmonary and hepatic metastases less than 12 months later.

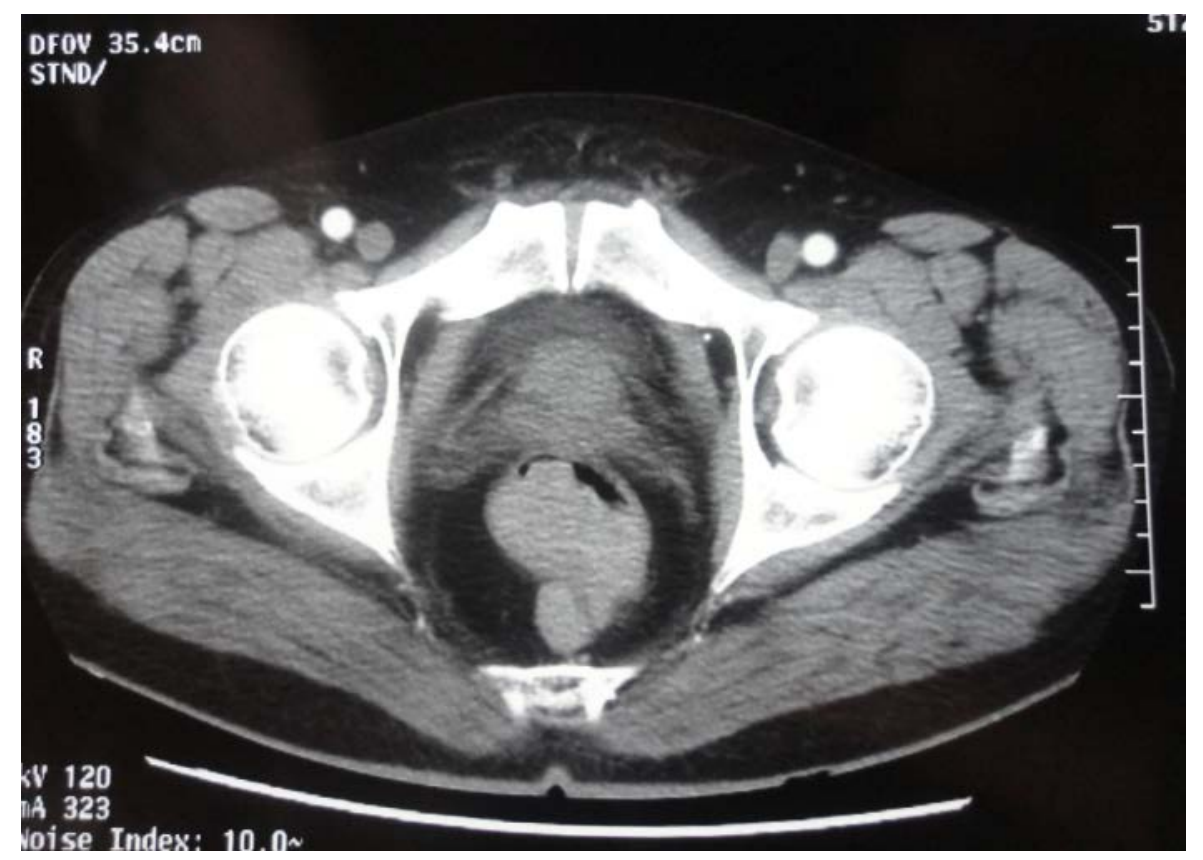

Figure 1. Pelvic MRI showing anorectal melanoma. 


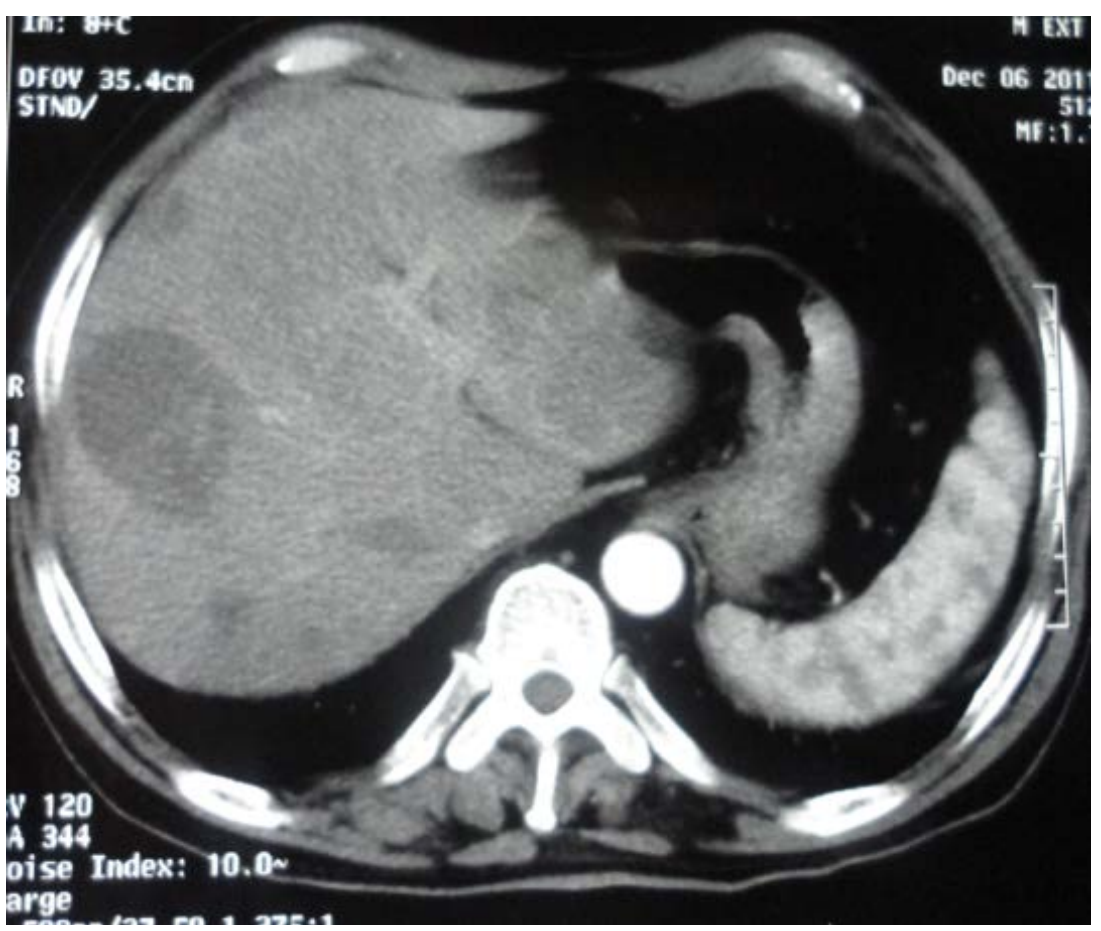

Figure 2. Radiological aspect of liver metastases of anorectal melanoma.

First line chemotherapy was received in all patients; it was based mainly on paclitaxel, carboplatine and dacarbazine. Response was modest with only 3 partial responses, 4 patients with disease stability, and 4 patients with disease progression. Two patients with over expression of C-KIT; received Imatinib as second line of treatment with significant improvement of symptoms and good radiological response reaching 50\%. Seven patients died with a median survival of 11 months from diagnosis to the date of death.

\section{Discussion}

The majority of colorectal and anal malignancies are adenocarcinomas and squamous cell cancers. Despite the predominance of these neoplasms at these locations, rare histotypes of the colon, rectum, and anus occur. These histotypes include lymphoma, melanoma, diffuse cavernous hemangioma, and sarcomas.

Primary anorectal melanoma is a very rare entity. About $1 \%$ of all anorectal tumors [4] [5]. Historically, melanocytes have been found within the transition zone beneath the dentate line and increases in number distally toward the anoderm [6] [7]. This has led to the presumption that anorectal melanoma arises from normal melanocytes distal to the dentate line that extend proximally into the rectum.

Regarding risk factors, sun exposure is not a risk factor for mucosal melanoma, and individuals with darker skin pigmentation have a higher incidence of anorectal melanoma. Interestingly, there is indirect evidence that infection with the human immunodeficiency virus (HIV) may increase the risk for development of anorectal melanoma [8]. This raises the question of an association between anorectal melanoma and immunosuppression, which will be addressed in the subsection on immunotherapy.

Typically presenting in the fifth or sixth decade of life with a slight predominance in woman.

Rectal bleeding is the most common presenting symptom followed by proctalgias and transit disorders [9].

Digital examination provides information concerning site, size, circumference, fixation and ulceration of the tumor. Proctosigmoidoscopy may be suggestive of anorectal melanoma when pigmentation is obvious. For anal canal melanomas present no specific clinical manifestations and due to their polypoid nature they are often misdiagnosed as a thrombosed hemorrhoid [10]. Obviously, the inexperienced endoscopist should always have this rare pathology in mind in order to avoid misdiagnosing this lesion as a thrombosed hemorrhoid.

Endoluminal ultrasound allows the evaluation of the tumor thickness and its' nodal status, but the diagnosis must always be based on the permanent sections due tendency of amelanotic types to masquerade as lymphoma, 
sarcoma or undifferentiated carcinoma [11].

Immunohistochemistry may also be helpful in the diagnosis of anorectal melanomas; S 100 protein, HMB-45, Melan A, and MiTF (microphthalmia-transcription-factor) are useful immunohistochemical markers.

In our patients, all biopsies had concluded to the diagnosis of melanoma after confirmation by immunochemistry with PS100, Melan A and HMB-45.

The KIT receptor tyrosine kinase plays an integral role in the normal development and function of melanocytes during embryonic and postnatal stages of growth. More recently, KIT aberrations have been associated with the pathogenesis of malignant melanoma.

KIT expression is typically detected in normal melanocytes (benign nevi and in situ melanomas), but it appears to be downregulated in the more invasive and metastatic forms of melanoma. A progressive decrease in KIT immunopositivity is associated with an increase in dermal invasiveness. It has been hypothesized that KIT signaling impacts the regulation of cell differentiation and tissue morphogenesis; thus, tumor progression may necessitate loss of KIT expression. In addition, reports of activating KIT mutations (and resultant protein overexpression) seen in a subset of melanoma patients point [12].

Regarding the extension of the disease, metastases occur via lymphatic and hematogenous routes and it has been reported that $38 \%$ of patients have already metastatic disease at the time of diagnosis [13]. Lymphatic spread to mesenteric nodes is more common than to inguinal nodes while lungs, liver and bones are the most frequent sites of distant metastases.

In the absence of any metastasis surgical therapy is indicated. Most series report no difference in survival in patients treated by wide local excision or abdominoperineal resection although the latter has proved more effective to control the local disease but, without clear improvement in survival [14] [15]. This is caused by the fact that most recurrences occur systemically regardless of the initial surgical procedure. However, a study suggests that sphincter-sparing local excision and adjuvant radiation is well tolerated and can effectively control local-regional disease while avoiding the functional morbidity of abdominoperineal resection [16]. The benefits of local excision are clear and include quicker recovery from a less invasive procedure, minimal impact on bowel function, and no need for a stoma. Prophylactic lymph node resection is of no value whereas therapeutic lymph node dissection is indicated only in the presence of positive inguinal nodes.

Radiation therapy also for local control has been mostly offered in the following two conditions, surgically non-resectable disease or as an adjuvant therapy in case of pathologically positive margins or positive lymphadenopathy.

For metastatic disease, the mean treatment is chemotherapy; its role in anorectal melanoma has not been completely defined because of the small number of cases. Dacarbazine has been considered the standard of treatment for metastatic or recurrent melanoma since 1972. Other useful antineoplasm agents include Interleukin-2, Temozolomide, Platinum analogs, nitrosoureas, and taxanes. The response rate of these single agents is $11 \mathrm{e} 22 \%$, with median overall survival of 5.6 - 11 months.

Results of our patients were consistent with literature's data, because they had mainly received Platinum, Taxanes and Dacarbazine with only 4 cases of partial response.

Regarding targeted therapies, only Vemurafenib and Ipilimumab had shown their efficacy in metastatic melanoma on phase III trials. But only Ipilimumab has been shown to improve overall survival. For Vemurafenib, approximately $40 \%$ to $60 \%$ of cutaneous melanomas carry mutations in BRAF V600E [17]. But mucosal melanomas have lower frequency of this mutation, comparing to other histological sub-types.

Melanoma is composed of several biologically distinct subtypes, each with unique genetic and clinical features, and each likely to respond differently to any one therapeutic strategy.

Melanoma arising from mucosal infrequently has BRAF mutations, but commonly has amplifications or activating mutations of KIT [18] [19].

KIT is an established therapeutic target in cancers with activating mutations of KIT, such as gastrointestinal stromale tumors (GIST), and significant benefit is achieved with various small molecule inhibitors of KIT including imatinib mesylate [20]. Several melanoma cell lines with KIT mutations are highly sensitive to imatinib mesylate [21] [22]. Furthermore, several patients with melanoma harboring KIT alterations, including a K642E mutation as well as a 7-codon duplication of exon 11, have been reported to achieve major durable responses to Imatinib mesylate [23] [24].

Two from our patients, with over expression of C-KIT had received Imatinib as a second line of treatment with good clinical response by improvement of performance statue and rectal symptoms. The radiological as- 
sessment had showed also a response reaching $50 \%$ on the primary tumors and metastases after 3 months of treatment.

That showed that new targeted therapies seem very hopeful with the identification of new biological targets.

\section{Conclusion}

Anorectal melanoma is a rare and aggressive cancer with high metastatic potential. The prognosis remains very poor for metastatic stage despite the systemic therapy. Treatment is still a big challenge and must be in a multidisciplinary approach. Results of targeting C-KIT with Imatinib were very hopeful and encouraging to develop new targets.

\section{References}

[1] Chang, A.E., Karnell, L.H. and Menck, H.R. (1998) The National Cancer Data Base Report on Cutaneous and Noncutaneous Melanoma: A Summary of 84,836 Cases from the Past Decade. Cancer, 83, 1664-1678. http://dx.doi.org/10.1002/(SICI)1097-0142(19981015)83:8<1664::AID-CNCR23>3.0.CO;2-G

[2] Balicevic, D., Tomic, K., Bekavac-Beslin 1., M., Kovacevic, I., Mijic, A., Belicza, M, et al. (2006) Synchronous Anorectal Melanoma. World Journal of Gastroenterology, 12, 3453-3455.

[3] van Schaik, P.M., Ernst, M.F., Meijer, H.A. and Bosscha, K. (2008) Melanoma of the Rectum: A Rare Entity. World Journal of Gastroenterology, 14, 1633-1635. http://dx.doi.org/10.3748/wjg.14.1633

[4] Roviello, F., Cioppa, T., Marrelli, D., Nastri, G., De Stefano, A., Hako, L. and Pinto, E. (2003) Primary Ano-Rectal Melanoma: Considerations on a Clinical Case and Review of the Literature. Chirurgia Italiana, 55, 575-580.

[5] Takahashi, T., Velasco, L., Zarate, X., Medina-Franco, H., Cortes, R., de la Garza, L. and Gamboa-Dominguez, A. (2004) Anorectal Melanoma: Report of Three Cases with Extended Follow-Up. Southern Medical Journal, 97, 311313. http://dx.doi.org/10.1097/01.SMJ.0000091034.77040.AC

[6] Walls, E.W. (1958) Observations on the Microscopic Anatomy of the Human Anal Canal. British Journal of Surgery, 45, 504-512. http://dx.doi.org/10.1002/bjs.18004519317

[7] Fenger, C. and Lyon, H. (1982) Endocrine Cells and Melanin-Containing Cells in the Anal Canal Epithelium. The Histochemical Journal, 14, 631-639. http://dx.doi.org/10.1007/BF01011895

[8] Cagir, B., Whiteford, M.H., Topham, A., Rakinic, J. and Fry, R.D. (1999) Changing Epidemiology of Anorectal Melanoma. Diseases of the Colon \& Rectum, 42, 1203-1208. http://dx.doi.org/10.1007/BF02238576

[9] Goldman, S., Babuche, G. and Pahlman, L. (1990) Anorectal Malignant Melanoma in Sweden: Report of 49 Patients. Diseases of the Colon \& Rectum, 33, 847-857. http://dx.doi.org/10.1007/BF02051925

[10] Winburn, G.B. (2001) Anal Carcinoma or “Just Hemorrhoids”? American Surgeon, 67, 1048-1058.

[11] Hillenbrand, A., Barth, T.F.E., Henne-Bruns, D. and Formentini, A. (2007) Anorectal Amelanotic Melanoma. Colorectal Disease, 10, 612-615. http://dx.doi.org/10.1111/j.1463-1318.2007.01400.X

[12] Antonescu, C.R., Busam, K.J., Francone, T.D., et al. (2007) L576P KIT Mutation in Anal Melanomas Correlates with KIT Protein Expression and Is Sensitive to Specific Kinase Inhibition. International Journal of Cancer, 121, 257-264. http://dx.doi.org/10.1002/ijc.22681

[13] Podnos, Y.D., Tsai, N.C., Smith, D. and Joshua, D.I. (2006) Factors Affecting Survival in Patients with Anal Melanoma. American Surgeon, 72, 917-920.

[14] Yeh, J.J., Shia, J., Hwu, W.J., Busam, K.J., Paty, P.B., Guillem, J.G., Coit, D.G., Wong, W.D. and Weiser, M.R. (2006) The Role of Abdominoperineal Resection as Surgical Therapy for Anorectal Melanoma. Annals of Surgery, 244, 10121017. http://dx.doi.org/10.1097/01.sla.0000225114.56565.f9

[15] Yap, L.B. and Neary, P. (2004) A Comparison of Wide Local Excision with Abdominoperineal Resection in Anorectal Melanoma. Melanoma Research, 14, 147-150. http://dx.doi.org/10.1097/00008390-200404000-00012

[16] Ballo, M.T., Gershenwald, J.E., Zagars, G.K., Lee, J.E., Mansfield, P.F., Strom, E.A., Bedikian, A.Y., Kim, K.B., Papadopoulos, N.E., Prieto, V.G. and Ross, M.I. (2002) Sphincter-Sparing Local Excision and Adjuvant Radiation for Anal-Rectal Melanoma. Journal of Clinical Oncology, 20, 4555-4558. http://dx.doi.org/10.1200/JCO.2002.03.002

[17] Davies, H., Bignell, G.R., Cox, C., et al. (2002) Mutations of the BRAF Gene in Human Cancer. Nature, 417, 949-954. http://dx.doi.org/10.1038/nature00766

[18] Curtin, J.A., Busam, K., Pinkel, D. and Bastian, B.C. (2006) Somatic Activation of KIT in Distinct Subtypes of Melanoma. Journal of Clinical Oncology, 24, 4340-4346. http://dx.doi.org/10.1200/JCO.2006.06.2984

[19] Beadling, C., Jacobson-Dunlop, E., Hodi, F.S., et al. (2008) KIT Gene Mutations and Copy Number in Melanoma 
Subtypes. Clinical Cancer Research, 14, 6821-6828.

[20] Demetri, G.D., von Mehren, M., Blanke, C.D., et al. (2002) Efficacy and Safety of Imatinib Mesylate in Advanced Gastrointestinal Stromal Tumors. The New England Journal of Medicine, 347, 472-480. http://dx.doi.org/10.1056/NEJMoa020461

[21] Antonescu, C.R., Busam, K.J., Francone, T.D., et al. (2007) L576P KIT Mutation in Anal Melanomas Correlates with KIT Protein Expression and Is Sensitive to Specific Kinase Inhibition. International Journal of Cancer, 121, 257-264. http://dx.doi.org/10.1002/ijc.22681

[22] Jiang, X., Zhou, J., Yuen, N.K., et al. (2008) Imatinib Targeting of KIT-Mutant Oncoprotein in Melanoma. Clinical Cancer Research, 14, 7726-7732. http://dx.doi.org/10.1158/1078-0432.CCR-08-1144

[23] Hodi, F.S., Friedlander, P., Corless, C.L., et al. (2008) Major Response to Imatinib Mesylate in KIT-Mutated Melanoma. Journal of Clinical Oncology, 26, 2046-2051. http://dx.doi.org/10.1200/JCO.2007.14.0707

[24] Lutzky, J., Bauer, J. and Bastian, B.C. (2008) Dose-Dependent, Complete Response to Imatinib of a Metastatic Mucosal Melanoma with a K642E KIT Mutation. Pigment Cell \& Melanoma Research, 21, 492-493.

http://dx.doi.org/10.1111/j.1755-148X.2008.00475.x 